\title{
Kompetenzen gezielt erwerben
}

\section{Zur Methodik Verhaltensorientierter Schulsozialarbeit}

FRANZ J. SCHERMER UND

MATHIAS BLANZ

Prof. Dr. Franz J. Schermer lehrt an der Hochschule für angewandte Wissenschaften Würzburg mit den Schwerpunkten Schulsozialarbeit, Verhaltensorientierte Handlungslehre und Psychologische Grundlagen der Sozialen Arbeit. Franz-Josef.Schermer@fhws.de

Prof. Dr. Mathias Blanz lehrt an der Hochschule für angewandte Wissenschaften Würzburg mit den Schwerpunkten Sozial-, Kommunikations- und Organisationspsychologie sowie Forschungsmethoden und Statistik.

Mathias.Blanz@fhws.de

\author{
Die Verhaltensorientierung stellt einen in Deutschland \\ bislang nicht etablierten Zugang in der Schulsozialarbeit \\ dar. Zu ihren Kennzeichen zählen das konkretisierende und \\ quantifizierende Vorgehen sowie die Evidenzbasierung. \\ Sie bietet einen empirisch ausgerichteten Zugang sowohl \\ für die Einzelfall- und Gruppenarbeit als auch für die \\ Arbeit mit Lehrern, Eltern und der Institution Schule.
}

Bei der Verhaltensorientierten Schulsozialarbeit handelt es sich um ein empirisch begründetes, also evidenzbasiertes Konzept der Sozialen Arbeit, aus dem ein umfangreicher Fundus wirksamer Methoden und Techniken abgeleitet werden kann (siehe Blanz \& Schermer, 2013; Schermer, Weber, Drinkmann \& Jungnitsch, 2006). Folgende Merkmale sind dabei konstitutiv:

(1) Das Konzept baut auf einem System empirisch bestätigter grundlagenwissenschaftlicher Gesetzmäßigkeiten aus unterschiedlichen erfahrungswissenschaftlichen Disziplinen auf. Hierbei kommt den Verhaltens- und Lerntheorien besondere Bedeutung zu.

(2) Es beinhaltet unterschiedliche, aus diesen Gesetzmäßigkeiten abgeleitete Methoden im Sinne von erfahrungswissenschaftlich fundierten (Regelbasierung), zielbezogenen (Indikation), strukturierten (Standardisierung), planmäßig eingesetzten (Planbarkeit) und wirksamen (Evaluation) Handlungsbegründungen.

(3) Es umfasst eine Reihe spezifischer Techniken als Handlungsanweisungen, die von den Methoden (widerspruchsfrei) abgeleitet sind und deren Funktionalität für die jeweilige Zielerreichung empirisch belegt ist.

(4) Es ist dem normativen Kontext der Sozialen Arbeit verpflichtet, der sich in der gesellschaftlichen Aufgabenstellung (z. B. Inklusionsver- mittlung, Exklusionsvermeidung und Exklusionsverwaltung), den rechtlichen Grundlagen (z. B. Sozialgesetzgebung) und berufsethischen Haltungen (z. B. Förderung von Menschenwürde, Menschenrechten und sozialer Gerechtigkeit) verdeutlicht (vgl. Como-Zipfel, 2013).

Die in der Schulsozialarbeit verfolgten Ziele (siehe z.B. Dupper, 2003; Franklin, Harris \& Allen-Meares 2006) erfordern unterschiedliche Arten von Methoden: Diagnostische Methoden beschäftigen sich mit der Bestimmung des Ist- und Sollzustandes des jeweiligen Anliegens sowie mit der Gestaltung der Arbeitsbeziehung, Interventionsmethoden beziehen sich auf den unmittelbaren Veränderungsprozess in Abhängigkeit von der jeweiligen Zielsetzung (edukativ, präventiv, korrektiv) und Evaluationsmethoden betreffen die Erfassung des Ausmaßes der Zielerreichung durch die jeweilige Maßnahme. Exemplarisch können mögliche verhaltensorientierte Interventionsmethoden und Interventionstechniken bei potentiellen Anliegen der Schulsozialarbeit aufgeführt werden (vgl. Abb. 1).

Das Vorgehen der Verhaltensorientierten Schulsozialarbeit soll im Folgenden unter Einbeziehung aller relevanten Bezugspersonen - Schüler, Lehrer, Eltern, Schule - anhand dreier typischer schulsozialarbeiterischer Aufgaben skizziert werden. 


\begin{tabular}{|l|c|c|}
\hline Anliegen & Interventionsmethoden & Techniken \\
\hline Angstbedingte Schulverweigerung & z. B. respondente Methoden & z. B. Entspannungstechniken \\
\hline Störverhalten im Unterricht (Zappelphilipp) & z. B. operante Methoden & $\begin{array}{c}\text { z. B. positive Verstärkung } \\
\text { (Lob, attraktive Tätigkeiten) }\end{array}$ \\
\hline Aufbau sozialer Kompetenz & z. B. Methoden des Modell- Lernens & $\begin{array}{c}\text { z. B. Rollenspiel (Rollentausch, } \\
\text { soziales Feedback) }\end{array}$ \\
\hline Gewalt in der Schule & z. B. Methoden des kognitiven Lernens & z. B. Instruktion / Regelaufbau \\
und -vermittlung
\end{tabular}

Abb. 1: Nach dem Konzept der Verhaltensorientierung können in der Schulsozialarbeit verschiedene Interventionsmethoden angewandt werden.

\section{Verhaltensorientierte Einzelfallarbeit: Funktionale Analyse von Schulverweigerung}

Verhaltensanalyse: Hier geht es darum, die aktuelle Form des schulverweigernden Verhaltens (V) multimodal zu beschreiben, zu quantifizieren und über die vorausgehenden $(S)$ und nachfolgenden $(\mathrm{K})$ Bedingungen zu erklären (siehe Abb. 2; vgl. Kearney \& Albano, 2007; Schermer, 2010). Die hierzu notwendigen Daten werden vom Schulsozialarbeiter im Gespräch mit den Betroffenen und über Beobachtung erhoben:

So geht der 10-jährige Andreas gar nicht mehr zum Unterricht. Am Morgen eines Schultags bleibt er zu lange im Bett, geht mehrmals auf die Toilette und übergibt sich, legt sich wieder hin (motorisch), klagt über Bauchschmerzen (physiologisch), äußert Angst vor der Schule (emotional) und glaubt, es nicht zu schaffen hinzugehen (kognitiv). Die Mutter schimpft und drängt ihn aufzustehen, versucht auch erfolglos ihn mit Spielen zu locken. Schließlich erlaubt sie ihm daheim zu bleiben. Diese Schwierigkeiten bestehen seit gut sieben Monaten, traten anfangs sporadisch (bei der Rückgabe von Proben), dann zunehmend gehäuft und in den letzten zwei Monaten an jedem Schultag auf. Die ersten Verweigerungen wurden beobachtet, nachdem im Unterricht während der Rückgabe einer Probe ein heftiger Feueralarm ausgelöst wurde, auf den Andreas mit Zittern und Weinen reagierte.

Das aktuelle Verhalten von Andreas lässt sich mit dem Prinzip der negativen Verstärkung erklären: Da die Mutter immer nachgibt, ist es ihm möglich, den Unterrichtsbesuch zu vermeiden. Die Entstehung des Problems kann durch respondents Lernen erklärt werden: Die durch den Feueralarm ausgelöste Angst assoziiert sich mit der Rückgabe von Proben und generalisiert auf die Schule.
Intervention und Evaluation: Zur Modifikation respondent verursachter Ängste stehen dem Schulsozialarbeiter zwei verhaltensorientierte Interventionsverfahren zur Verfügung, die bei Andreas zur Anwendung kamen: Schrittweise Annäherung durch Verhaltensübungen (Bewältigung des Schulwegs, Aufsuchen des Schulgebäudes, Teilnahme an ausgewählten Unterrichtstunden, vollständiger Schulbesuch etc.) sowie muskuläre Entspannung nach Jacobson, d. h. Andreas lernte einen seiner Angst entgegengesetzten Zustand selbst herbeizuführen und damit der Angst entgegenzuwirken. Zur Förderung von Motivation und der Festigung des Erreichten wurde aus den operanten Methoden die Technik der differentiellen Verstärkung eingesetzt, d. h. Andreas erhielt von den Bezugspersonen für die erfolgreiche Durchführung der Übungen eine Belohnung.

Mithilfe des geschilderten Vorgehens der Schulsozialarbeit konnte binnen sechs Wochen der vollständige und kontinuierlich anhaltende (letzte Katamnese nach zwei Jahren) Schulbesuch erreicht werden.

\section{Verhaltensorientierte Gruppenarbeit: Training sozialer Kompetenzen}

Das Gruppentraining sozialer Kompetenzen (GsK) von Hinsch \& Pfingsten (2007) wendet sich auch an Kinder und Jugendliche mit Schwierigkeiten im sozialen Bereich, wie beispielsweise selbstunsichere oder ängstliche Schüler. Für die Schulsozialarbeit steht dabei der präventive Charakter des Trainings zur Erhaltung und Förderung der psychosozialen Gesundheit der Schüler im Vordergrund.

Verhaltensanalytische Modellvorstellungen: Das Prozessmodell sozial inkompetenten Verhaltens des Gruppentrainings sozialer Kompetenzen kann bildhaft dargestellt werden (vgl. Abb. 3). Auslöser für das selbstunsichere Verhalten sind oft Situationen mit Anforderungscharakter, beispielsweise wenn bei dem Schüler persönliche Ziele - »sich beschweren wollen « - mit sozialen Zielen - "gemocht werden wollen « - zusammentreffen. Beim selbstunsicheren Verhalten finden sich häufig Überzeugungen, die Situation nicht bewältigen zu können (kognitiv), Gefühle wie

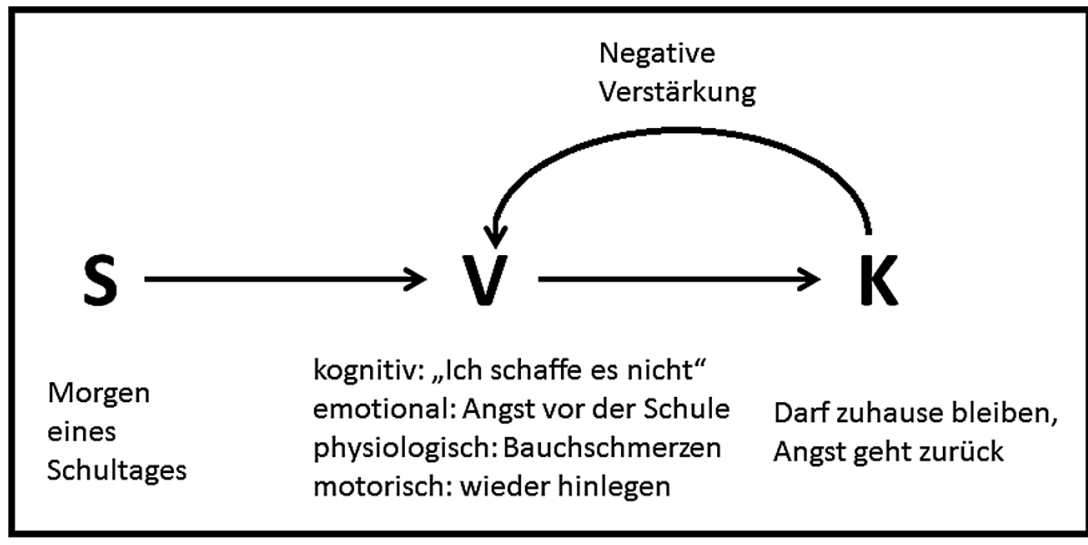

Abb. 2: Das Prinzip der negativen Verstärkung kann für Erklärungen bei Schulproblemen dienen. 


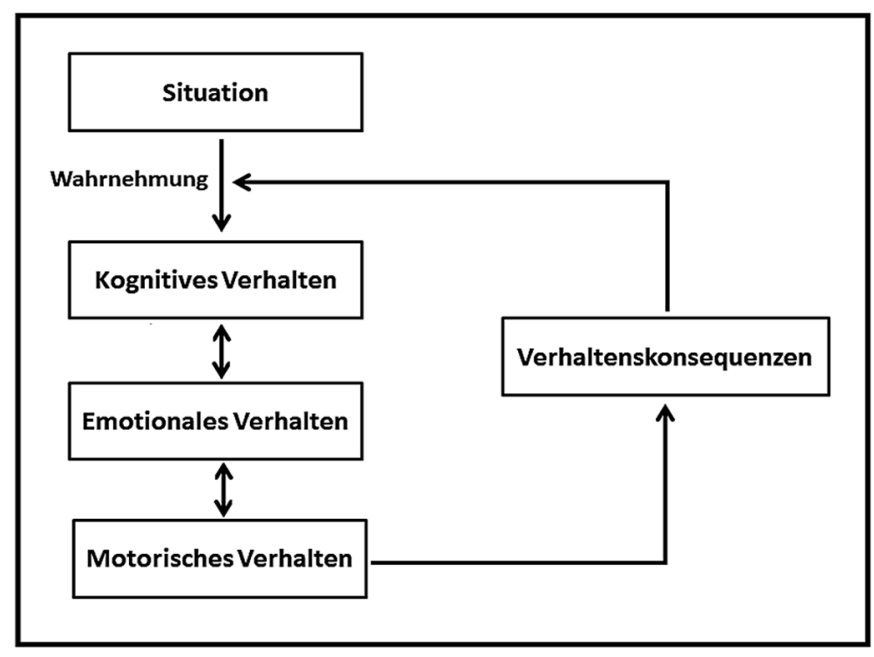

Abb. 3: Sozial inkompetenten Verhaltensweisen liegen oft Überzeugungen zugrunde, eine Situation nicht bewältigen zu können.

Mutlosigkeit (emotional) und entweder Vermeidungsverhalten oder Verhaltensdefizite (motorisch) wie beispielsweise keinen Blickkontakt halten oder zu leises Sprechen. Die negativen Konsequenzen dieses Verhaltens tragen zu seiner Aufrechterhaltung bei (vgl. Abb. 3).

Intervention und Evaluation: Im Rahmen von Rollenspielen werden zu den genannten Situationstypen (vgl. Abb. 4) Anforderungssituation in Kleingruppen eingeübt. Dabei stehen positive Verstärkung und Verhaltensaufbau im Vordergrund. Die Dauer des Trainings beträgt rund sieben Sitzungen zu je zweieinhalb Stunden, zwischen denen die Lernfortschritte in der Praxis umgesetzt werden. Die Wirksamkeit des Trainings wurde in mehreren Studien belegt. Bei selbstunsicheren Klienten zeigte sich beispielsweise eine Reduktion von Kritik- und Misserfolgsangst sowie eine Steigerung von Fordern-Können und von Neinsagen-Können, die über eineinhalb Jahre anhielten.

\section{Verhaltensorientierte Arbeit mit der Institution Schule: Gewaltprävention nach Olweus}

Verhaltensanalytische Modellvorstellungen: Im »Teufelskreismodell des Bullying " von Olweus (2006) wird die Entwicklung des gewalttätigen Verhaltens als ein Lernprozess verstanden, der sich vor allem aus dem Einfluss von Verstärkung (operantes Lernen) und Beobachtung (Modell-Lernen) ergibt (vgl. Abb. 5). Dabei wird eine Wechselwirkung zwischen den folgenden intra- und interindividuellen Faktoren angenommen: a) wehrlos-passives Hinnehmen der Übergriffe durch die Opfer, b) zuschauend-duldendes Verhalten der Mitschüler, c) Unentschlossenheit und fehlendes Eingreifen der Lehrkräfte und d) Nichtinformiertheit der Eltern.

Intervention und Evaluation: Das Kernprogramm besteht aus verschiedenen Maßnahmen, die auf den Ebenen Schule, Klasse, Schüler und Eltern ansetzen. Auf Schulebene wird zunächst im Rahmen eines sogenannten pädagogischen Tages, an dem Schulleitung, alle Lehrkräfte, Schulsozialarbeiter, ein externer Experte sowie je ein Vertreter des Schüler- und Elternbeirates teilnehmen, ein individueller Handlungsplan entwickelt, der aus den Ergebnissen einer Datenerhebung abgeleitet wird. Zur Förderung des Problembewusstseins und der Akzeptanz für die geplanten Maßnahmen wird anschließend eine Schulkonferenz durchgeführt, auf der durch einen formellen Beschluss der Verpflichtung und Verantwortung der Schule für die Ziele offiziell Ausdruck verliehen wird. Dazu zählt auch, dass die Aufsichten auf dem Schulhof und während des Mittagessens verstärkt werden. Auf Klassenebene steht die Einführung von Klassenregeln im Vordergrund (Beispiel: »Wir werden andere Schüler nicht mobben" oder "Wir werden Schülern, die gemobbt werden, helfen«). Die Regelbefolgung wird operant unterstützt durch ein Klassengespräch mit festem wöchentlichem Termin, auf dem die Umsetzung der Regeln besprochen wird und Verstöße gegegenfalls sanktioniert werden. Auf persönlicher Ebene schließlich werden mit Tätern und Opfern individuelle Gespräche geführt, ebenso mit den Eltern der beteiligten Schüler.

Zur Evaluation des Olweus-Programms werden Prä-Post-Fragebögen verwendet, die von Schulsozialarbeitern leicht eingesetzt werden können. Nahezu weltweit liegen mittlerweile Evaluationsdaten vor, teilweise mit über mehrere Jahre reichenden Katamnesen, die eine deutliche Wirksamkeit des Programms belegen: Bei richtiger und vollständiger Implementierung werden Angaben zwischen 50 und 70 Prozent für den Gesamtbereich antisozialen Verhaltens berichtet. In einer in Deutschland (Schleswig-Holstein) durchgeführten Studie liegen die Angaben etwas niedriger (Hanewinkel \& Knaack, 2004).

\section{Resümee und Ausblick}

Die Verhaltensorientierung betont die Verhaltensaneignung als Hauptkriterium für eine erfolgreiche Schulsozialarbeit (vgl. Speck 2006; 2009). Dies dient einer Professionalisierung der Schulsozialarbeit im Sinne einer Aufwertung ihres Stellenwertes. Das häufig thematisierte ganzheitliche Vorgehen bedeutet in der Verhaltensorientierten Schulsozi- 


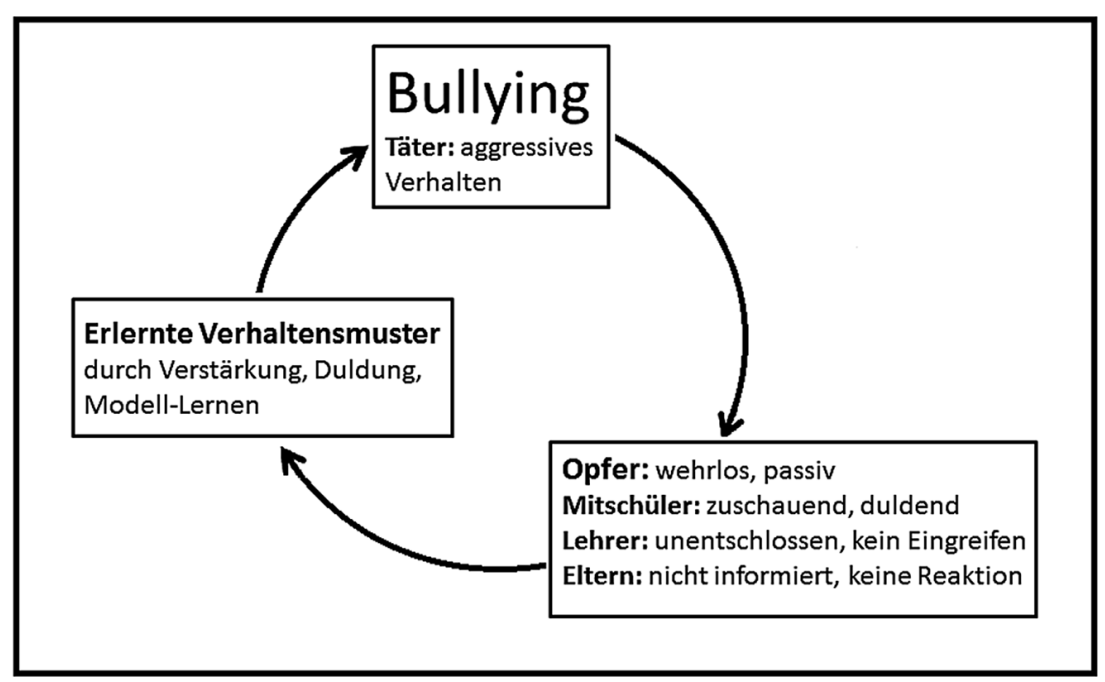

Abb. 5: Das Mobbing unter Kindern und Jugendlichen wird oft als "Bullying" bezeichnet. Durch falsches Verhalten der Beteiligten kann dabei leicht ein Teufelskreis entstehen.

alarbeit, dass alle wesentlichen Faktoren berücksichtigt werden müssen, die zur Aufrechterhaltung eines Anliegens beitragen. Es ist aus unserer Sicht zu wünschen, dass evidenzbasierte Methoden im Rahmen der Ausbildung in Sozialer Arbeit an Hochschulen auch in Deutschland zunehmend an Bedeutung gewinnen. Gerade die Universalität des verhaltensorientierten Ansatzes kommt der Schulsozialarbeit mit ihren heterogenen Aufgabenbereichen entgegen.

\section{Literatur}

Blanz, M., Como-Zipfel, F. \& Schermer, F. J. (Hg.) (2013). Verhaltensorientierte Soziale Arbeit. Grundlagen, Methoden, Handlungsfelder. Stuttgart: Kohlhammer.

Blanz, M. \& Schermer, F. J. (2013). Methoden der Verhaltensorientierten Sozialen Arbeit. In M. Blanz, F. Como-Zipfel \& F. J. Schermer (Hg.), Verhaltensorientierte Soziale Arbeit. Grundlagen, Methoden, Handlungsfelder, S. 63-102. Stuttgart: Kohlhammer.

Como, F. (2013). Wissenschaftshistorische und berufsethische Grundlagen der Verhaltensorientierten Sozialen Arbeit. In M. Blanz, F. Como-Zipfel \& F. J. Schermer (Hg.), Verhaltensorientierte Soziale Arbeit. Grundlagen, Methoden, Handlungsfelder, S. 13-33. Stuttgart: Kohlhammer.

Dupper, D.R. (2003). School Social Work. Skills and Interventions for effective Practice. Hoboken, N. J.: John Wiley \& Sons.

Franklin, C., Harris, M.B. \& Allen-Meares, P. (Eds.) (2006). The School Services Sourcebook.
A Guide for School-Based Professionals. Oxford: Oxford University Press.

Hanewinkel, R. \& Knaack, R. (2004). Prävention von Aggression und Gewalt an Schulen. Ergebnisse einer Interventionsstudie. In H. G. Holtappels, W. Heitmayer, W. Melzer \& K.-J.

Tillmann (Hg.), Forschung über Gewalt an

Schulen. Erscheinungsformen und Ursachen, Konzepte und Prävention, S. 299-314. 3. Aufl. Weinheim: Juventa.

Hinsch, R. \& Pfingsten, U. (2007). Das Gruppentraining sozialer Kompetenzen (GsK). Grundlagen, Durchführung, Materialien. 5.

Kearney, C.A. \& Albano, A.M. (2007). When children refuse school: A cognitive-behavioral therapy approach/Therapist guide. New York: Oxford University Press.

Olweus, D. (2006). Gewalt in der Schule. Was Lehrer und Eltern wissen sollten und tun können. 2. Aufl. Bern: Huber Verlag.

Schermer, F. J. (2010). Verhaltensdiagnostik und Verhaltensmodifikation bei schulverweigerndem Verhalten. In A. Reichart, G. Adams, N. Beck, M. Holler \& A. Warnke (Hg.), Der Schüler mit psychischer Störung in der Betreuung von Kinder- und Jugendpsychiatrie, Jugendhilfe und Schule, S. 21-45. Lengenrich: Papst.

Schermer, F.J., Weber, A., Drinkmann, A. \& Jungnitsch, G. (2005). Methoden der Verhaltensänderung: Basisstrategien. Stuttgart: Kohlhammer.

Speck, K. (2006). Qualität und Evaluation in der Schulsozialarbeit. Konzepte, Rahmenbedingungen und Wirkungen. Wiesbaden: VS Verlag für Sozialwissenschaften.

Speck, K. (2009). Schulsozialarbeit. Eine Einführung. 2. Aufl. München: Ernst Reinhardt. Aufl. Weinheim: PVU.

\section{Leistungspflicht Gesetzlicher Krankenversicherungen}

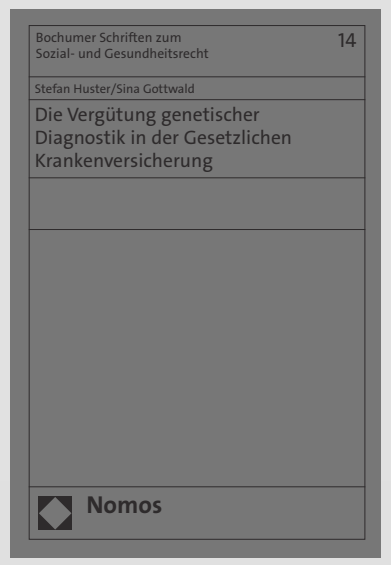

\section{Die Vergütung genetischer Diagnostik in der Gesetzlichen Krankenversicherung Von Prof. Dr. Stefan Huster und RAin Sina Gottwald 2013, 72 S., brosch., 19,- $€$ ISBN 978-3-8487-0400-2 (Bochumer Schriften zum Sozial- und Gesundheitsrecht, Bd. 14)}

Genetische Untersuchungen spielen für die medizinische Diagnostik eine immer größere Rolle. Für die Gesetzliche Krankenversicherung stellt sich damit die Frage, ob und inwieweit diese Untersuchungen von ihrer Leistungspflicht erfasst werden. Dieser Band untersucht diese Frage und konzentriert sich dabei auf die Entwicklungen in der Personalisierten Medizin.

www.nomos-shop.de/20782

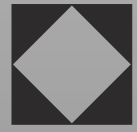

Nomos 